\title{
Cellular and genetic signatures of operational tolerance in kidney transplant recipients through single cell RNA sequencing analysis
}

\author{
Hyunjoo Bae ${ }^{1}$, Hanbi Lee ${ }^{2}$, Ji Hyeong Ryu ${ }^{1}$, Joo Hee Jang ${ }^{3}$, Jihyun Lee ${ }^{3}$, Geon Young Ko ${ }^{3}$, Chul Woo Yang ${ }^{2}$, \\ Byung Ha Chung ${ }^{2}$, Eun-Jee $\mathrm{Oh}^{1}$

\footnotetext{
${ }^{1}$ Department of Laboratory Medicine, The Catholic University of Korea, Seoul St. Mary's Hospital, Seoul, Korea

${ }^{2}$ Department of Internal Medicine-Nephrology, Seoul St. Mary's Hospital, College of Medicine, The Catholic University of Korea, Seoul, Korea

${ }^{3}$ Department of Biomedical Science, The Catholic University of Korea, Seoul St. Mary's Hospital, Seoul, Korea
}

Background: Patients with operational tolerance do not use immunosuppressants after renal transplantation, but they show stable post-transplant results as stable patients with immunosuppressants. We analyzed differently expressed mRNAs and proteins by targeting immune genes to single cells in patients with rejection, stable, and tolerance after kidney transplantation to see if the post-kidney transplantation stability could be distinguished from genetic signatures.

Methods: This experiment was conducted with peripheral blood mononuclear cells (PBMC) of four post-transplant rejection patients, four post-transplant stable patients, and five post-transplant tolerance patients. Through targeted multi-omic analysis, 10,180 (rejection group), 7,180 (stable group), and 16,784 (tolerance group) single-cell transcriptomes were analyzed. We added 20 different types of ab-seq to the targeted panel to complement for the relationship between proteins and transcripts.

Results: We found 17 subclusters in the PBMC samples of three groups and confirmed the expression of mRNA and protein targeted by the immune panel among the subpopulations. We found the difference in the expression level of each group of NK cells, CD4 T cells, CD8 T cells, B cells, Treg cells, B memory cells and B naive cell populations. In 420 target genes including 20 Ab-seq, 70 transcripts and proteins were expressed differently in rejection and stable, 45 in stable and tolerance, and 96 differently in rejection and tolerance. Compared with the other two groups of patients, in tolerance patients, CD56(Ab) was highly expressed in B cells, CD4 T cells, and Treg cells, and CD196(Ab) was highly expressed in B memory cells and B naive cells. CD8(Ab) was highly expressed in NK cells.

Conclusions: Analysis of transcript expression at the single cell level characterizes the phenotype of cells and defines their functional properties. We found that the operational tolerance group expressed markers that differed from the rejection and stable group.

Corresponding author: Eun-Jee Oh

E-mail: ejoh@catholic.ac.kr

(c) The Korean Society for Transplantation
This is an Open Access article distributed under the terms of the Creative Commons Attribution Non-Commercial License (http://creativecommons.org/li-
censes/by-nc/4.0/) which permits unrestricted non-commercial use, distribution, and reproduction in any medium, provided the original work is properly cited. 